\title{
Interferometric Remapped Array Nulling
}

\author{
F. Vakili ${ }^{1}$, E. Aristidi ${ }^{1}$, L. Abe ${ }^{1}$, and B. Lopez ${ }^{2}$ \\ ${ }^{1}$ Laboratoire Univ. d'Astroph. de Nice (LUAN), CNRS UMR 6525, Parc Valrose, 06108 Nice Cedex 02, France \\ 2 Laboratoire Fresnel, CNRS UMR 6528, Observatoire de la Côte d'Azur, BP 4229, 06304 Nice cedex 4, France
}

Received 4 September 2003 / Accepted 20 March 2004

\begin{abstract}
This paper describes a method of beam-combination in the so-called hypertelescope imaging technique recently introduced by Labeyrie in optical interferometry. The method we propose is an alternative to the Michelson pupil reconfiguration that suffers from the loss of the classical object-image convolution relation. From elementary theory of Fourier optics we demonstrate that this problem can be solved by reconfiguring images instead of pupils. Imaging is performed in a combined pupil-plane where the point-source intensity distribution (PSID by comparison to the more commonly quoted point-spread function, PSF) tends towards a pseudo Airy disc for a sufficiently large number of telescopes. Our method is applicable to snap-shot imaging of extended sources with a field limited to the Airy pattern of single telescopes operated in a co-phased multi-aperture interferometric array. It thus allows to apply conveniently pupil plane coronagraphy. Our technique called Interferometric Remapped Array Nulling (IRAN) is particularly suitable for high dynamic imaging of extra-solar planetary companions or extra-galactic objects where long baseline interferometry would closely probe the central regions of AGNs for instance. We also discuss the application of IRAN to improve the performances of imaging and/or nulling interferometers like the full-fledged VLTI array or the DARWIN space-borne mission.
\end{abstract}

Key words. techniques: high angular resolution - techniques: interferometric

\section{Introduction}

Modern optical interferometry is among the most promising techniques to directly detect and characterize extra-solar planets (ExPN) using the so-called nulling (Bracewell 1978) or differential interferometry (Petrov et al. 2000). Nulling means to reject the light of the on-axis star to detect its planet otherwise enshrouded by the star diffracted light. The technique is now being effectively considered on large telescope groundbased arrays like the VLTI-GENIE (Gondoin et al. 2003) among others or space-borne missions such as DARWIN (Mennesson \& Mariotti 1997) or TPF (Beichman et al. 2002). Quite recently, alternative techniques such as apodized apertures (Soummer et al. 2003; Gonsalves \& Nisenson 2003) or coronagraphic densified arrays of telescopes, also called hypertelescopes (Labeyrie et al. 2003), have been proposed to achieve the same goal with the bonus of offering imaging possibilities up to the diffraction limit set by their baseline.

Compared to Bracewell nulling interferometry, the concept of densified pupil-hypertelescope presents in principle the additional advantage of separating the ExPN energy from the emission of its parent star. The principle of hypertelescope is a generalization of the classical Michelson periscopic setup for stellar interferometry (Michelson 1920). Therefore and

Send offprint requests to: F. Vakili, e-mail: Farrokh.Vakili@unice.fr compared to the co-axial combination considered by nulling interferometers, densified-pupil hypertelescopes should be more immune from the exo-zodiacal contamination inherent to coaxial pupil-plane detection techniques used for DARWIN or TPF missions. On the other hand, in a densified-pupil the basic relation of image formation from the convolution of the PSID to sky brightness degrades as a function of input versus output baselines normalized to the geometric telescope size. Thus the non-aberrated imaging field of view of a hypertelescope can dramatically decrease with increasing densification factor $\gamma$ (Gillet et al. 2003). As already noted by Labeyrie and in absence of optical aberrations this field, also called Zero-Order-Field (ZOF), becomes intrinsically small and attains a small fraction of the primary field of the elementary telescopes that form the interferometric array. The advantage of a densified optical array of telescopes equipped by an imageplane phase coronagraph over a nulling long baseline interferometer remains an open question that several groups have studied or are studying both for theoretical and laboratory prototyping aspects (Traub 1986; Pedretti et al. 2000).

In the followings we propose a pupil-plane imaging technique which is equivalent to Labeyrie's hypertelescope technique with the extra bonus of conserving the convolution relation over a flat-field limited to the super-imposed pupilla of the primary telescopes. In the followings we first schematically describe the principle of our technique called Interferometric 

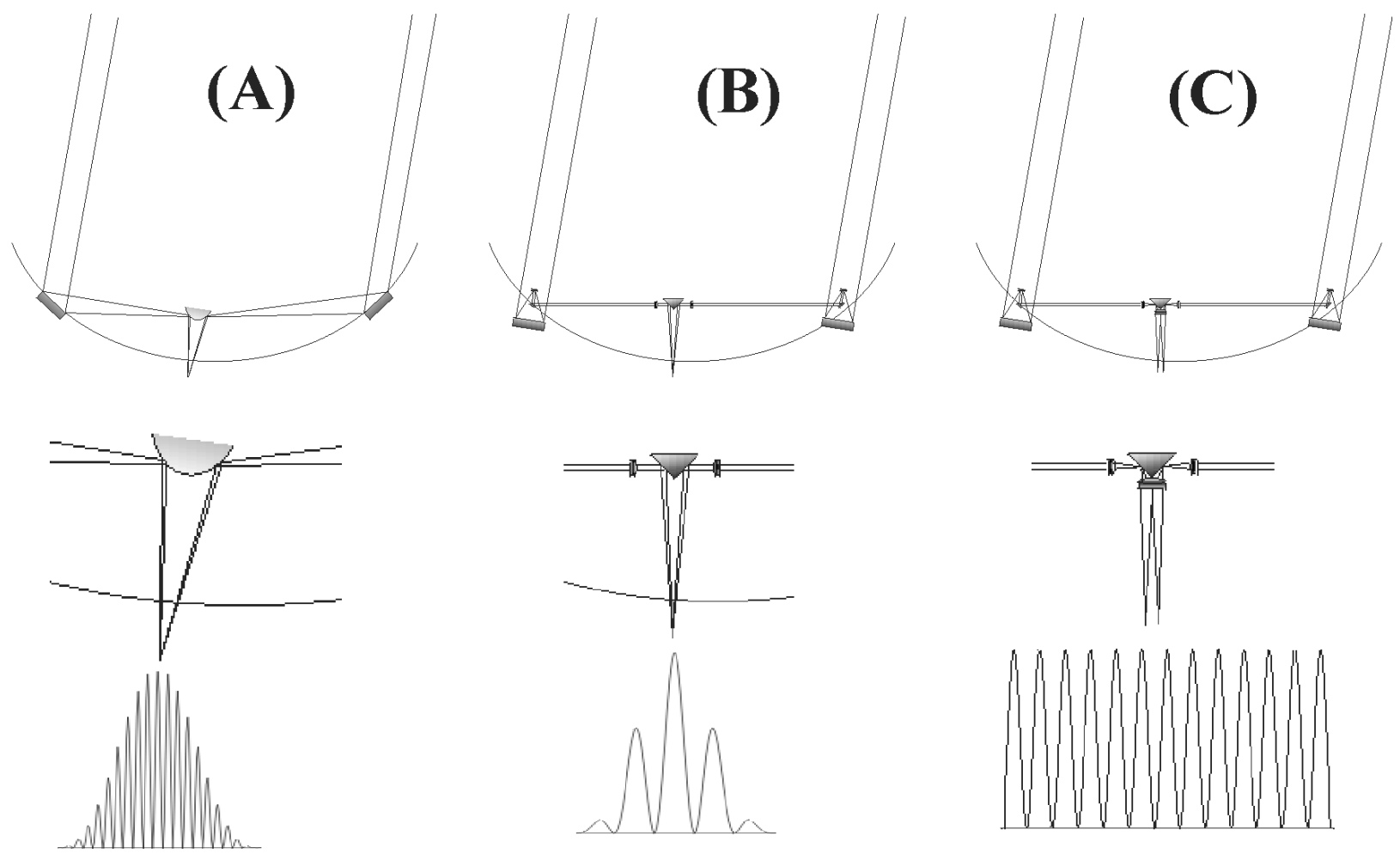

Fig. 1. Generic comparison of three different beam-combinations for an optical stellar interferometer. (A) and (B) the classical Fizeau versus Michelson beam-combinations, $(\mathrm{C})$ instead of superimposing the Airy patterns from the telescopes it is possible to use a relay lens after the beam-combiner so as to stack the two output pupils on the top of each other with a modulation depending on the output Airy discs distance. This pupil-plane interferometry can indeed be generalized to $\mathrm{N}$ telescopes described as IRAN beam-combination described in Sect. 2.2.

Remapped-Array Nulling (IRAN), establish formally its operation and describe its various properties through numerical simulations. Two different optical set-ups to practically implement the IRAN technique making use of a nulling coronagraph for ExPN detection are also outlined. Finally the application of IRAN to ground-based as well as space-borne missions is discussed.

\section{Principle of IRAN}

Labeyrie's hypertelescope concept is based on densifying the output pupil of an interferometric array by conserving the primary telescope orientations respective to each other to form the equivalent of a single dish telescope with a continuous surface. This is obtained for instance by re-imaging the output pupils on a pyramidal beam combiner (Gillet et al. 2003). The resulting diffraction pattern obtained from the pseudo-single densified aperture will correspond to an Airy pattern if the sub-apertures were to be co-phased, thus resembling to a monolithic giant dish Airy pattern. In the IRAN concept beam-combination is simply obtained by forming output images from primary telescopes on the same pyramid as for the hypertelescope (Fig. 1). A relay lens is then used to stack all the output pupils on the top of each other and record their interference on a 2D detector.

\subsection{Technical implementation}

To better understand the operating principle of IRAN it is useful to recall the academic Fizeau versus Michelson optical setups for stellar interferometry. In a Fizeau-type beam combination (Fig. 1A) the light beams from segments of a giant primary mirror are focused by a secondary Cassegrain mirror to form a fringe pattern which modulates the refolded Airy discs. Both these patterns undergo the same angular magnification which depends on the mirror segments size and their spacing. In general the more distant the segments the more fringes across the Airy pattern. On the contrary in the Michelson set-up (Fig. 1B) the fringe modulation does not depend on the spacing between the input telescope pupil size but on the output pupils as seen from the focal superimposed Airy patterns. In the Michelson set-up the basic convolution relation between the Point Spread Function and the object intensity distribution on the sky is lost making image reconstruction from the measure of the complex visibility function mandatory. In both cases the fringe intensity pattern is modulated by the Airy envelope. The Michelson set-up can be further modified (Chelli \& Mariotti 1986) to form the Airy discs on the faces of the beam-combination mirror followed by a relay lens (Fig. 1C) which would form two superimposed and cosine-modulated output pupils where the fringe period depends on the Airy disc pattern distance as seen from the two superimposed pupils. In this case the fringe modulation remains constant across the support of the superimposed pupils. Now if much more than 2 Airy patterns were remapped 
from a large number of input mirror segments the different period and orientation of the resulting cosine fringe modulations will produce a central bright spot at the center of the conjugate stacked pupil for an on-axis star.

Two beam-combination schemes could be envisaged in this case: a classical bulky optical pyramidal shape mirror (Rousselet-Perraut et al. 1997) which generalizes (Fig. 1C) set-up versus a fiber optics (FO) beam combiner (Mariotti et al. 1996) with the bonus of modal filtering and an expected simplified beam-combiner. In the case of FO combination, the field of view would be limited to the Airy angular size of individual telescopes.

\subsection{Intensity distribution for an on axis point-source}

For sake of simplicity we consider a horizontal array of optical telescopes spread over co-centric circles with increasing radii and number of telescopes per circle. The telescopes afocal beams feed a central beam-combiner (Fig. 1C) after the constantly changing optical paths between the telescopes are corrected by optical delay lines for the sideral motion of the object. Note that the projected interference pattern at the focal plane of the interferometer will also change due to the sideral motion. This will correspond to an anamorphic change of the object brightness spatial sampling in the zenithal direction but its exact treatment is beyond the scope of the present study and will be addressed in a next paper.

\subsubsection{Expression of the monochromatic PSID}

Let $\boldsymbol{R}_{i}$ the positions of the $N$ telescopes on the ground. These telescopes are supposed identical, with diameter $d_{0}$ and focal length $f_{0}$. Each telescope produces an Airy pattern of diameter $2.44 \lambda f_{0} / d_{0}$ in its focal plane. We suppose that the incoming light is monochromatic with a wavelength of $\lambda$.

Beam-collection from the $N$ telescopes of the array is done after $N$ field-lenses (individual diameter $d_{1}$ and focal length $f_{1}$ ) will form corresponding Airy patterns on the $N$ reflecting faces of a pyramidal beam combiner or by feeding bundled FO. The collected - and not the combined - images in the common plane $P_{1}$ Fig. 2) are centered at positions $\boldsymbol{\rho}_{i}$. The geometry of these images replicates the input telescopes pupil orientation so that

$\boldsymbol{R}_{i}=\gamma \boldsymbol{\rho}_{i}$

where $\gamma$ is the scaling factor between the two planes of input pupils versus output Airy discs. To fix further ideas, we chose a telescope configuration distributed over 3 rings of diameters $D_{1}, D_{2}=2.4 D_{1}$ and $D_{3}=3.8 D_{1} .7$ telescopes are equally distributed on the first ring, 13 on the second one and 19 on the third one. This configuration is non-redundant in order to minimize the energy spread in the secondary peaks of the PSID. All the figures shown hereafter, except Sect. 4 about coronography, correspond to numerical simulations made with the configuration which follows:

- wavelength $\lambda=10 \mu \mathrm{m}$;

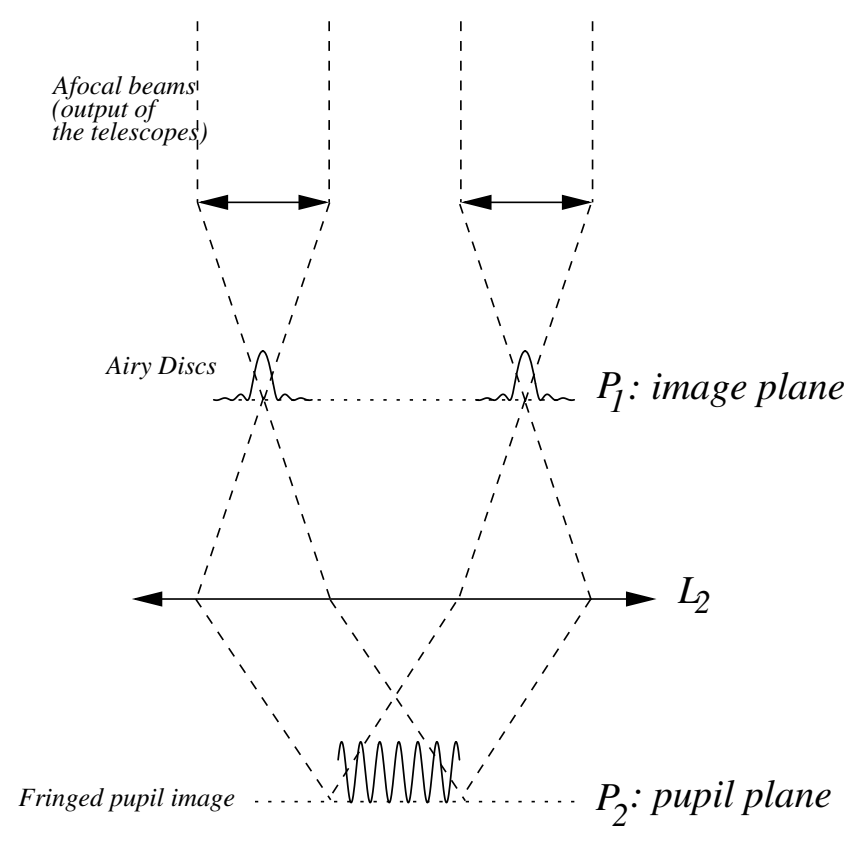

Fig. 2. Optical layout of the IRAN beam-combiner. Afocal beams coming from the telescopes are focused on the image plane $P_{1}$ where several Airy discs are observed (here only two of them are shown). A common lens $L_{2}$ produces a fringed pupil image in the pupil plane $P_{2}$.

- diameter of the telescope circles: $D_{1}=20 \mathrm{~m}, D_{2}=48 \mathrm{~m}$, $D_{3}=76 \mathrm{~m}$;

- $d_{1}=1 \mathrm{~cm}, f_{1}=10 \mathrm{~cm}$, no fiber optics are used for afocal beam transportation;

- the Airy discs are spread over 3 circular rings of radii $r_{1}=$ $1, r_{2}=2.4$ and $r_{3}=3.8$ in units of $2.44 \lambda f_{1} / d_{1}$. This gives $\gamma=4.1 \times 10^{4}$.

The complex amplitude of the light in the plane $P_{1}$ (Fig. 2) is given by:

$\psi_{1}(\boldsymbol{\rho})=A(\boldsymbol{\rho}) * \sum_{i=1}^{N} \delta\left(\boldsymbol{\rho}-\boldsymbol{\rho}_{i}\right)$

where $\rho$ is the position vector in the plane and $\delta()$ is the Dirac delta distribution. $A(\rho)$ is the amplitude distribution of the images given by the lenses focusing each afocal beam. For primary circular apertures $A(\rho)$ will be the amplitude of an Airy pattern of diameter $2.44 \lambda f_{1} / d_{1}$ if the light is transported by classical optics (mirrors) from the main telescope focus. In the case of optical fibers used for beam transportationcombination, $A(\rho)$ would rather become a Gaussian function.

Figure 3 depicts the intensity distribution in the plane $P_{1}$ for our experimental setup.

A lens $L_{2}$ of diameter $d_{2}$ and focal length $f_{2}$ is placed after $P_{1}$ and produces an image of the pupil in its focal plane $P_{2}$ containing the interferences between the $N$ beams. The amplitude in the plane $P_{2}$ is given by the Fourier transform of $\psi_{1}$ (Goodman 1996):

$\psi_{2}(\boldsymbol{r})=P(\boldsymbol{r}) . \sum_{i=1}^{N} \exp -\frac{2 i \pi \boldsymbol{r} . \boldsymbol{\rho}_{i}}{\lambda f_{2}}$ 


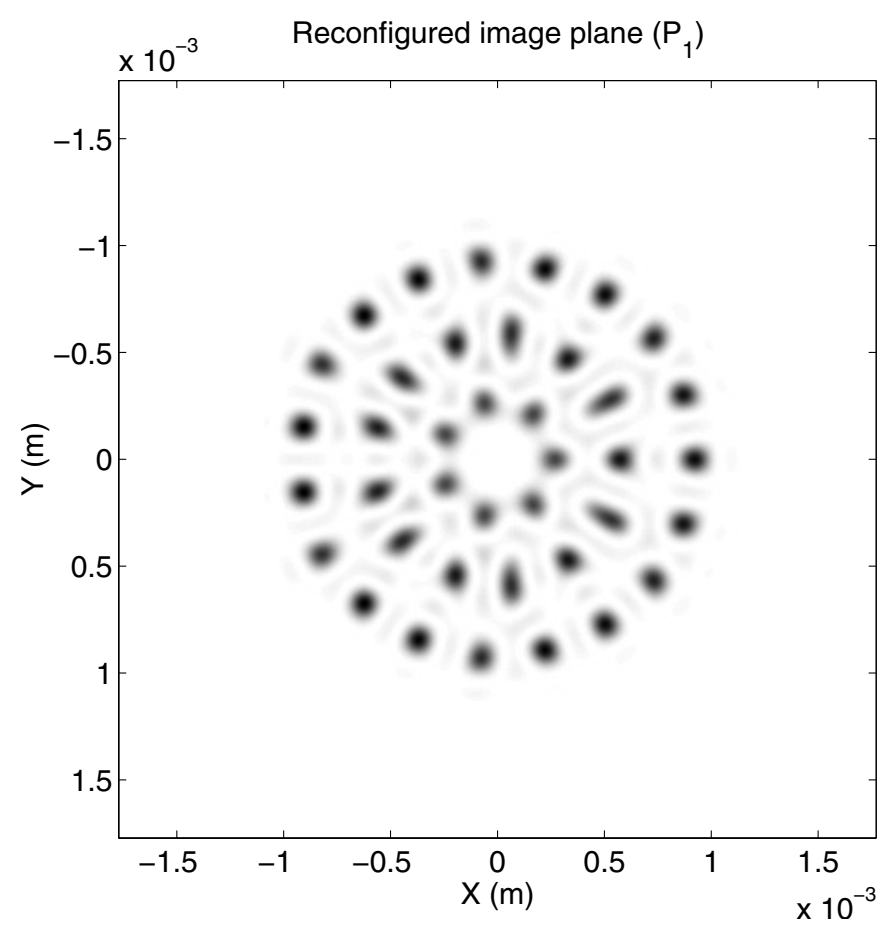

Fig. 3. Simulation of the configuration of the Airy discs in the plane $P_{1}$ for a interferometer with $N=39$ primary telescopes (gray-level plot of the intensity). Experimental setup is described in the text. The units on both axii are in meter in the focal plane $P_{1}$. As the diffraction patterns from individual telescopes are coherent between each-other, they interfere in amplitude if they get too close in the plane $P_{1}$. Therefore in that case the individual Airy patterns apparently deform but this does not hamper the PSID image in the final pupil plane $P_{2}$.

where $P(\boldsymbol{r})$ is the Fourier transform of $A(\boldsymbol{\rho})$. In the case of bulk optics $P(r)$ is the re-imaged telescope pupil identical to $P_{2}$. The corresponding intensity is

$I_{2}(\boldsymbol{r})=|P(\boldsymbol{r})|^{2} \cdot\left|\sum_{i=1}^{N} \exp -\frac{2 i \pi \boldsymbol{r} . \boldsymbol{\rho}_{i}}{\lambda f_{2}}\right|^{2}$.

\subsubsection{Description of the PSID and of the field of view}

The function $|P(\boldsymbol{r})|^{2}$ is the achromatic pupil function. As for $A(\rho)$, its shape depends on the instrumental setup (bulk optics or optical fiber combination). It is ideally a uniform disc of diameter $d_{1} f_{2} / f_{1}$. This function will constitute the physical limit of the field of view (FOV) of the interferometer ${ }^{1}$. The other term, denoted as

$I_{0}(\boldsymbol{r})=\left|\sum_{i=1}^{N} \exp -\frac{2 i \pi \boldsymbol{r} \cdot \boldsymbol{\rho}_{i}}{\lambda f_{2}}\right|^{2}$

is a complex interference pattern which exhibits a pseudo Airy disc at the center of the super-imposed pupils with several rings. Figure 5 shows the simulation of the PSID in the plane $P_{2}$

1 This is a consequence of the existence of an object-image convolution relation demonstrated in Sect. 2.4, allowing to convert a position in the focal plane into an angle on the sky. Therefore the spatial extent of the PSID will limit the field of view. for the experimental configuration described above. The radial cut taken along the $x$-axis is displayed in Fig. 6. It can be seen that the size of the central spot is that of an Airy from an optical aperture of $D_{3}$ in size. Therefore the angular resolution Res of the interferometer is conserved by the IRAN beam-combination

$R e s \simeq \frac{\lambda}{D_{3}}$.

For our present simulation this resolution corresponds to $\operatorname{Re} s=$ 0.027 arcsec.

It can also be seen in Fig. 5 that at some distance from the centre of the simulated PSID secondary maxima start to crowd the FOV with a fixed pseudo-speckle background. This noise is due to the filling gaps of energy in the intermediate image plane $P_{1}$ and becomes noticeable at an angular distance of $\lambda / D_{1}$ from the center (see Fig. 6). This distance is exactly the angular resolution of a telescope of diameter $D_{1}$.

The total FOV of the interferometer is given by the function $|P(\boldsymbol{r})|^{2}$. In the ideal case where this pupil function equals to 1 within a circular area of diameter $d_{1}$ (diameter of the afocal beams) the function $|P(\boldsymbol{r})|^{2}$ is a uniform disc of diameter $d_{1} f_{2} / f_{1}$. At the boundary of the pupil, i.e. $\boldsymbol{r}=d_{1} f_{2} /\left(2 f_{1}\right)$, using Eq. (17) one obtains the final FOV, expressed in arcs, as

$F_{t}=\frac{d_{1}}{\gamma f_{1}}$

In this ideal case the FOV depends only upon the scaling factor $\gamma$ and the aperture ratio of the lenses at the output of the fibers. For our example we obtain a total FOV of 0.5 arcsec but this can theoretically be as large as desired within the limits of optics geometrical aberrations. On the other hand in the case of fiber-optics beam-combination, the shape of the function $|P(\boldsymbol{r})|^{2}$ will become Gaussian with a FWHM determined by the numerical aperture of the optical fiber (Ruilier 1999).

The PSID of our experimental setup presents a "clean" aspect within a FOV similar to the Airy disc of a telescope of diameter $D_{1}(0.12$ arcsec for the case of our simulation). In this clean zone, the PSID exhibits secondary rings of amplitude less than $1 / 100$ of the maximum. Therefore we can define a clean $F O V$ of radius

$F_{\mathrm{c}} \simeq \frac{\lambda}{D_{1}}$

within which direct imagery is possible with contrast conditions comparable to the PSF of a monolithic telescope. The reason why the intensity of the secondary maxima in our 39 telescope array is smaller than those of a classical Airy pattern disc is that the radii of the three circles on which telescope diffraction images spread have been optimized to concentrate the central intensity of the PSID and to obtain a clean FOV as low as possible (see Fig. 4).

\subsubsection{Effect of a finite bandwith}

The PSID expression $I_{2}(\boldsymbol{r})$ is given in Eq. (4). It is the product of two terms. One of them is achromatic $\left(|P(\boldsymbol{r})|^{2}\right)$ and the other is $\lambda$-dependent. Figure 7 displays the distribution of $I_{2}(r)$ for 


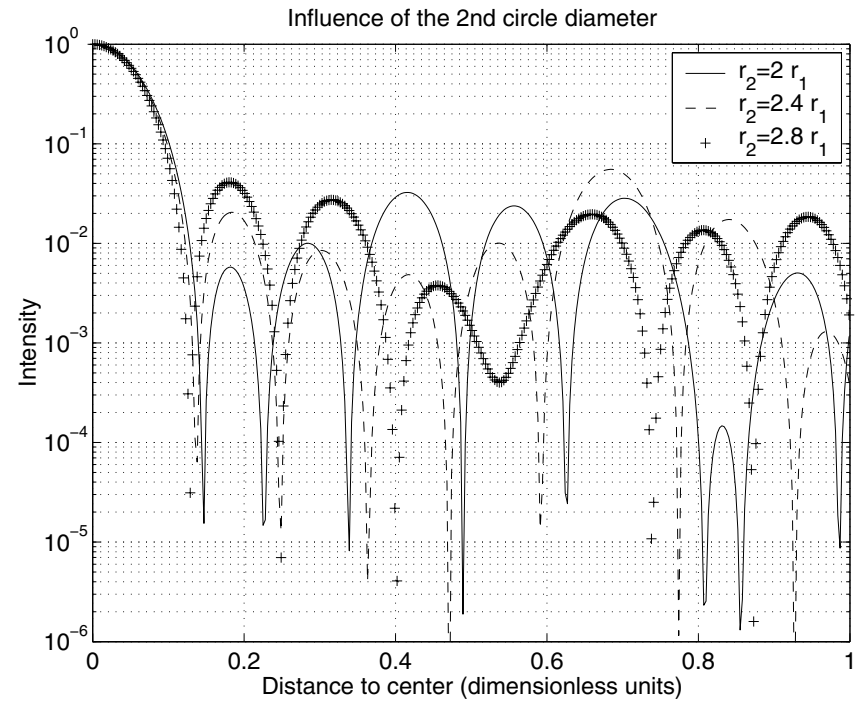

Fig. 4. Radial cuts of the intensity distribution of the IRAN PSID for an array of $N=39$ telescopes. The values of the radii $r_{1}, r_{2}$ and $r_{3}$ of the 3 inner to the outer rings have been set to minimize the secondary maxima inside the clean FOV.

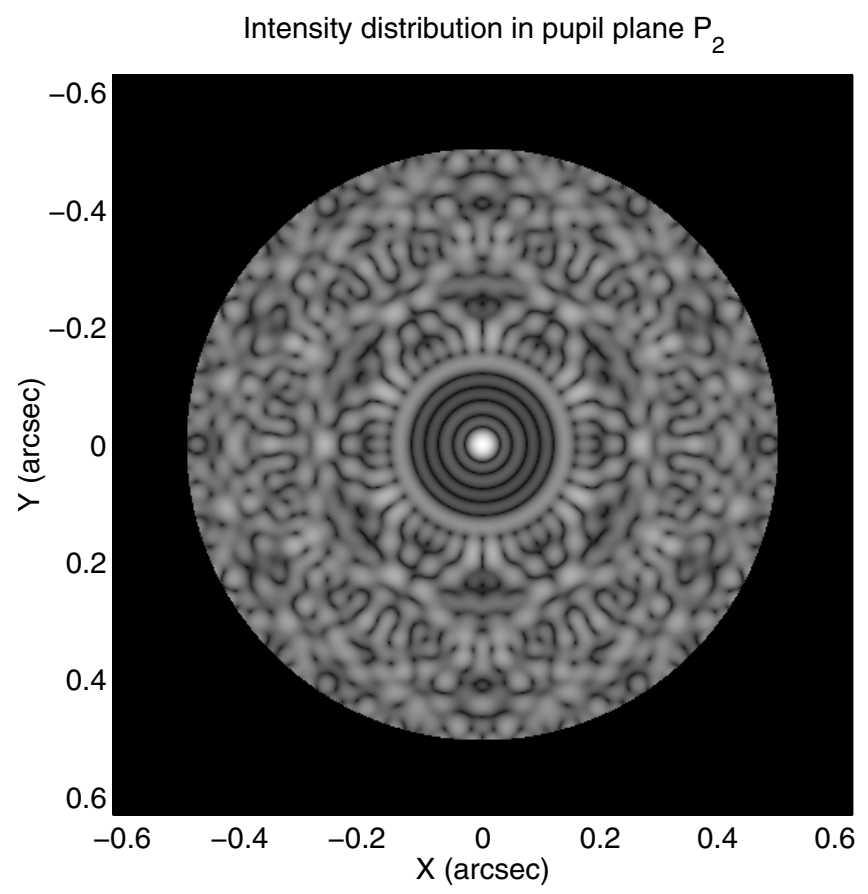

Fig. 5. Simulation of the intensity pattern $I_{2}(\boldsymbol{r})$, also called PSID (see the text), in the pupil plane $P_{2}$ for a interferometer with $N=39$ telescopes observing an on-axis point-source. The wavelength is $\lambda=$ $10 \mu \mathrm{m}$. Experimental configuration is described in the text. A pseudo Airy disc is visible at the center of the pupil image. Axis units have been converted into arcsec according to Eq. (17).

three values of $\lambda$. It can been seen that the total FOV given by the width of $|P(\boldsymbol{r})|^{2}$ does not depend on $\lambda$. However inside this FOV the structures are scaled as a function of wavelength. For a finite bandwith $\Delta \lambda$ the intensity in the plane $P_{2}$ for an on-axis point-source results from an integral over the wavelength $\lambda$ :

$I_{\Delta \lambda}(\boldsymbol{r})=\int_{\Delta \lambda} f(\lambda) I_{2}(\boldsymbol{r} ; \lambda) \mathrm{d} \lambda$

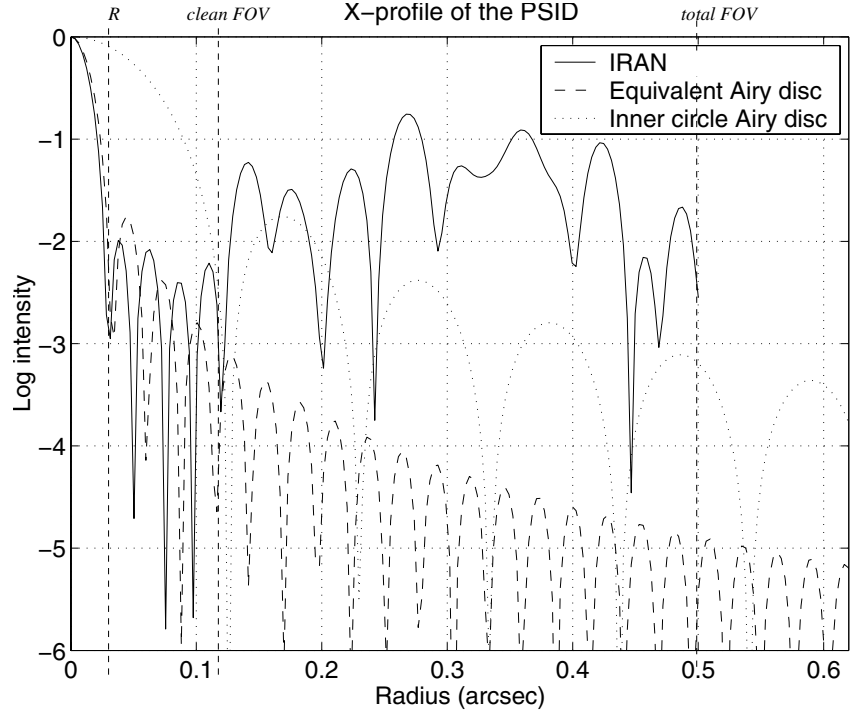

Fig. 6. Radial cut of the monochromatic PSID in the pupil plane $P_{2}$. The experimental configuration is described in the text. This cut is compared to that of the Airy disc from a single dish monolithic telescope: dashed line is for an aperture diameter $D_{3}$ (the outer circle of telescopes), dotted line is for an aperture diameter $D_{1}$ (inner circles of telescopes). Vertical dashed lines give the interferometer resolution $R$, the clean FOV and the total FOV.

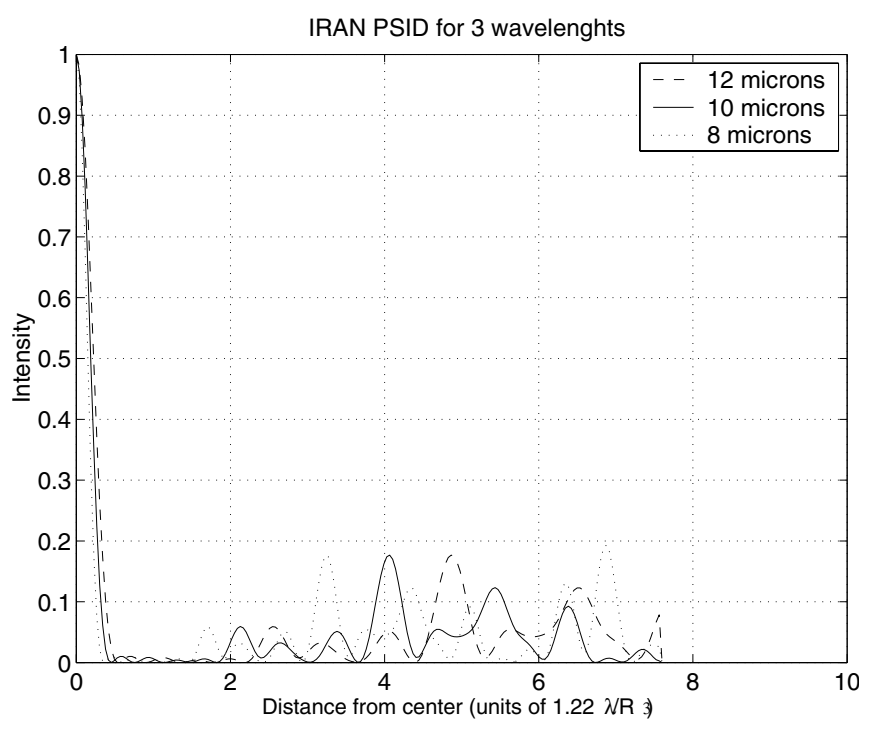

Fig. 7. Radial cut of the PSID for tree different wavelengths, taken along the $x$-axis. The simulation Parameters are described in the text. $x$-axis has been labelled in units of $1.22 \lambda_{0} / D_{3}$, i.e. the angular resolution of the interferometer at $\lambda_{0}=10 \mu \mathrm{m}$.

where $f(\lambda)$ is the product of the spectrum of the incident light by the spectral transmission of the interferometer. Figure 8 displays the intensity pattern $I_{\Delta \lambda}(\boldsymbol{r})$ for a central wavelength $\lambda_{0}=10 \mu \mathrm{m}$ and for different values of $\Delta \lambda$. The function $f(\lambda)$ has been taken to be the unity. It turns out that the pseudospeckle structures of secondary maxima disperse as radial spectra. This dispersion forces these pseudo-speckles to become a unifrom background with increasing distance from the center of the FOV. 

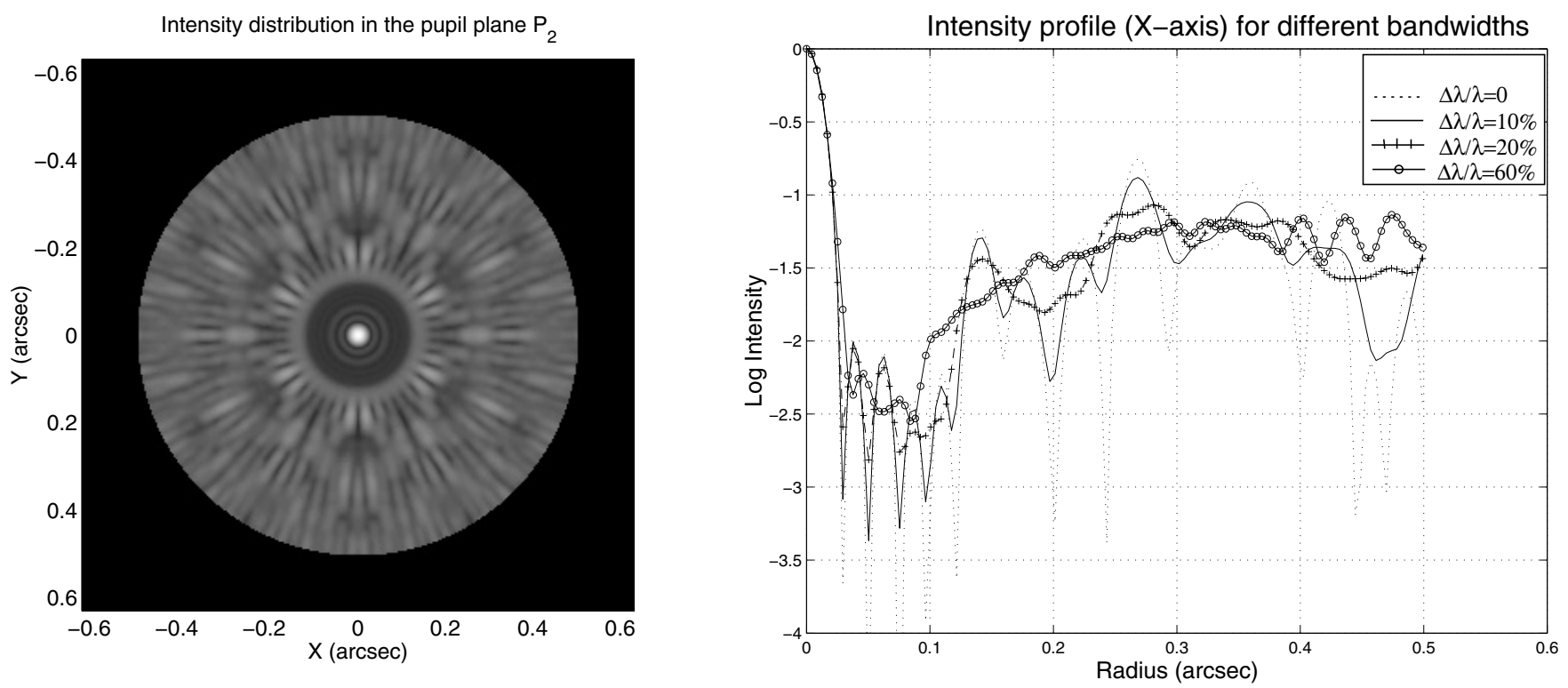

Fig. 8. Left: gray-level plot of the PSID for a central wavelength of $10 \mu \mathrm{m}$ and a bandwidth $\Delta \lambda=2 \mu \mathrm{m}$. Right: radial cut of the same PSID taken along the $x$-axis for different bandwidths. Note that the center of the PSID has been thresholded so as the secondary maxima can be better depicted.

\subsection{The response for an off-axis point-source}

We observe an off-axis point source in the direction given by the vector $\boldsymbol{\theta}=(\alpha, \delta)$ where $\alpha$ (resp. $\delta$ ) is the offset in right ascension (resp. declination). The images at the focus of each telescope are then shifted by a vector $\boldsymbol{\Delta}=f_{0} \boldsymbol{\theta}$. We assume that the angle between the axis and the source is small enough so that the shift $|\Delta|$ remains small in comparison to the size of the Airy disc. If the light is collected by an optical fiber, there is an attenuation factor on the complex amplitude denoted as $g(\boldsymbol{\theta})$.

The difference with the on-axis configuration is a piston term depending on the telescopes position

$p_{i}=\exp \frac{2 i \pi \theta \cdot \boldsymbol{R}_{i}}{\lambda}$

This piston term will conserve after beam combination. The complex amplitude of the light in the image plane $P_{1}$ becomes

$\psi_{1}(\boldsymbol{\rho})=g(\boldsymbol{\theta}) A(\boldsymbol{\rho}) * \sum_{i=1}^{N} p_{i} \delta\left(\boldsymbol{\rho}-\boldsymbol{\rho}_{i}\right)$

and in the pupil plane $P_{2}$ :

$\psi_{2}(\boldsymbol{r})=g(\boldsymbol{\theta}) P(\boldsymbol{r}) . \sum_{i=1}^{N} \exp -\frac{2 i \pi\left(\boldsymbol{r}-\gamma f_{2} \boldsymbol{\theta}\right) \cdot \boldsymbol{\rho}_{i}}{\lambda f_{2}}$.

The corresponding intensity is

$I_{2}(\boldsymbol{r})=g(\boldsymbol{\theta})^{2}|P(\boldsymbol{r})|^{2} . I_{0}\left(\boldsymbol{r}-\gamma f_{2} \boldsymbol{\theta}\right)$.

As for the on-axis case, the term $|P(\boldsymbol{r})|^{2}$ is a geometrical limitation of the field in the pupil plane. Within this limitation, the function $I_{2}(\boldsymbol{r})$ appears to be almost invariant by translation, as long as $g(\boldsymbol{\theta})$ remains close to 1 , e.g. for small values of $|\boldsymbol{\theta}|$.

\subsection{Object-image relation}

We consider now an object composed of $M$ point-sources having each-one an intensity of $O_{i}$ at the position $\boldsymbol{\theta}_{i}$ from the center of the field of view. We assume $\left|\boldsymbol{\theta}_{i}\right|$ small enough so that the image of the object at the telescope focal plane is well inside the telescope Airy disc. The above relations give the intensity in the pupil plane $P_{2}$ :

$I_{2}(\boldsymbol{r})=|P(\boldsymbol{r})|^{2} \sum_{i=1}^{M} O_{i} g\left(\boldsymbol{\theta}_{i}\right)^{2} I_{0}\left(\boldsymbol{r}-\gamma f_{2} \boldsymbol{\theta}_{i}\right)$.

Finally we consider the general case of on object of brightness distribution $O(\boldsymbol{\theta})$. Making the assumption that the object is small enough to be fully contained within the Airy disc of each telescope, the above relation generalizes into:

$I_{2}(\boldsymbol{r})=|P(\boldsymbol{r})|^{2} \iint O(\boldsymbol{\theta}) \mathrm{d}^{2} \theta g(\boldsymbol{\theta})^{2} I_{0}\left(\boldsymbol{r}-\gamma f_{2} \boldsymbol{\theta}\right)$.

We introduce here the function

$O^{\prime}(\boldsymbol{\theta})=O(\boldsymbol{\theta}) g(\boldsymbol{\theta})^{2}$

that represents the brightness distribution of the object weighted by the acceptance function of the optical fiber if necessary. The intensity in the pupil plane expresses as

$I_{2}(\boldsymbol{r})=|P(\boldsymbol{r})|^{2} I_{0}(\boldsymbol{r}) * \iint O^{\prime}(\boldsymbol{\theta}) \delta\left(\boldsymbol{r}-\gamma f_{2} \boldsymbol{\theta}\right) \mathrm{d}^{2} \theta$

and finally

$I_{2}(\boldsymbol{r})=\frac{1}{\left(\gamma f_{2}\right)^{2}}|P(\boldsymbol{r})|^{2}\left[I_{0}(\boldsymbol{r}) * O^{\prime}\left(\frac{\boldsymbol{r}}{\gamma f_{2}}\right)\right]$.

Inside the boundaries delimited by the pupil function $|P(\boldsymbol{r})|^{2}$, we find the classical convolution relation between the PSID and the object (again multiplied by the acceptance function of the optical fiber if necessary) scaled by the factor $\gamma f_{2}$. This factor allows to convert a position $x$ in meters in the focal plane into an angle $\theta=x /\left(\gamma f_{2}\right)$ in radian on the sky. 


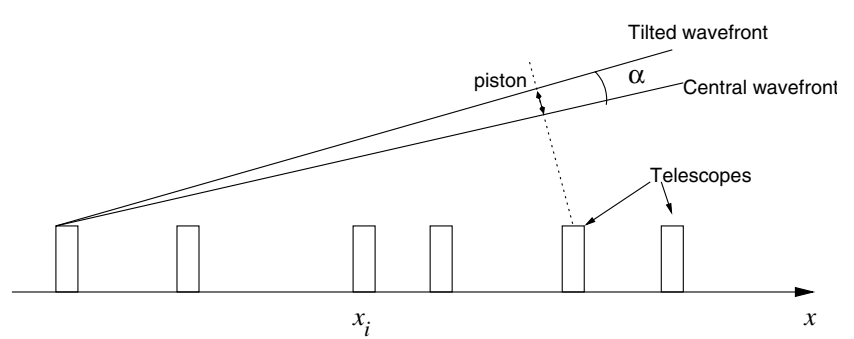

Fig. 9. Simulated observation of an off-axis point-source at a distance $\alpha$ from the center of the field of view. On this one-dimensionnal configuration, each telescope is at a position $x_{i}$. The corresponding complex amplitude is multiplied by a piston term $\exp 2 i \pi \alpha x_{i} / \lambda$.

\section{Direct images of stellar companions}

The object-image convolution relation of Eq. (17) is an interesting property for imagery at the interferometer resolution. In the simple case of a double star, the focal image is the sum of two PSIDs at a distance corresponding to the star and its companion separation times the magnification factor and weighted by their intensity ratio.

Figures 10a,b show a numerical simulation of a double star monochromatic image with an angular separation of 50 mas and a magnitude difference of 3 (the setup for this simulation is the 3-ring configuration described in Sect. 2.2 operating at the wavelength $\lambda=10 \mu \mathrm{m}$ ). The angular separation taken so that the companion image forms inside the "clean zone" described in Sect. 2.2 where the direct detection is more simple.

When the angular separation of the binary increases, the noise from the pseudo-speckle background of the central star can make the detection of the companion more difficult, as illustrated by Figs. 10c,d where the companion image falls on the dirty zone of the main star intensity pattern.

Equation (17) shows that the companion image is at the same position in $P_{2}$ whatever the wavelength. Increasing the bandwidth, in addition of collected number of photons from the companion can be a mean to improve the detection SNR since the dispersion of the pseudo-speckles turns out to smooth their noise. Figures 10e,f depict the numerical simulation of a binary star image with a separation of 300 mas, a magnitude difference of 3 and a relative bandwidth of $\Delta \lambda / \lambda=60 \%$.

\section{Coronography}

Detecting very faint companions around a star becomes an optical challenge with the increasing magnitude difference. For a ExPN such as 51 Pegb this difference is of the order of 7 in $N$-band. Various coronographic techniques have been proposed (Soummer et al. 2003, and references therein) to reject the energy of the on-axis star. The Achromatic Interfero Coronograph (AIC) (Gay \& Rabbia 1996) appears as particularly suitable for ExPN detection with the IRAN interferometric configuration which is operated in the pupil plane. The principle of the AIC is summarized in Fig. 11. A lens $L_{3}$ relays the pupil plane $P_{2}$ to form a geometric image of the remapped Airy discs in the $P_{1}^{\prime}$ plane. A classical laboratory Michelson interferometer is placed between $L_{3}$ and $P_{1}^{\prime}$. A lens plus a cat's eye optical system is introduced into one of the arms of this interferometer to produce a $\pi$ phase-shift and rotating the beam by $180^{\circ}$. In $P_{1}^{\prime}$ a total nulling of the light incoming from an on-axis source is observed if the complex amplitude in $P_{1}$ is a pair function, i.e. for a symmetric telescopes configuration. Finally the $L_{4}$ lens focuses the light coronagraphic image of the pupil plane $P_{2}^{\prime}$, on the top of the detector. For an off-axis point-source, twin images are obtained inside this image.

A numerical simulation has been performed in monochromatic light with a symmetric telescope configuration composed of 36 apertures spread over 3 rings (as in the previous section). We put 6 equally-spaced telescopes on the first ring, 12 on the second and 18 on the outer ring. The external diameter is $76 \mathrm{~m}$, the wavelength is $10 \mu \mathrm{m}$. Corresponding image plane $P_{1}$ displays a set of 36 Airy discs with the same geometry: in particular the complex amplitude is a pair function. The observed PSIDs with and without coronography are compared in Fig. 12 for an on-axis source. Indeed for a perfect wavefront the nulling effect is total (note that the residual "noise" is due to the roundoff errors from computer simulations).

Double star simulations are shown in Fig. 13 for two different separations between the components: a small separation of 10 mas (to be compared to the interferometer resolution of 30 mas) and a large separation of 200 mas where the companion falls inside the "dirty" zone of the main star's image. It can be seen that in both cases the secondary companion can be easily detected. Note that for this simulation the magnitude difference is chosen to be 5 , but since the on axis star is fully nulled one would detect ExPNs for any magnitude difference for a perfect wavefront through the whole atmosphere and interferometer+AIC -coronagraph optics. Therefore technical set-up and atmospherical conditions will be the only limitation to our proposed nulling concept. A study of the AIC performances can be found in Baudoz et al. (Baudoz et al. 2000a,b). Our densifiedimage combination and its coupling to AIC for IRAN need to be studied closely in details but are beyond the scope of the present paper.

\section{Discussion}

Optical aperture synthesis in optical wavelengths can be considered as a mature observing technique at the present. Its effective application to detect exo-planetary systems or to image extended sources with high contrast still requires a number of conceptual and technological difficulties to be overcome. The IRAN concept developed in this paper gives to our knowledge the first formal description and generic set-ups to fulfill this goal. The advantage of using a diluted array over a large monolithic mirror, assuming the primary telescopes were mobile across the interferometric array (like the VLA radio interferometer), is that the angular resolution of the interferometric array could be adaptively changed to match the angular separation of a star and its companion. A Fizeau-type is not however optimum in terms of sensitivity because the coherent energy dilutes among more and more fringes with expanding baselines. The alternative pairwise beam-combination is on the other hand inefficient when a very large number of sub-apertures were to be recombined. All-in-one combination of a large number of sub-pupils using IRAN approach is attractive because the 

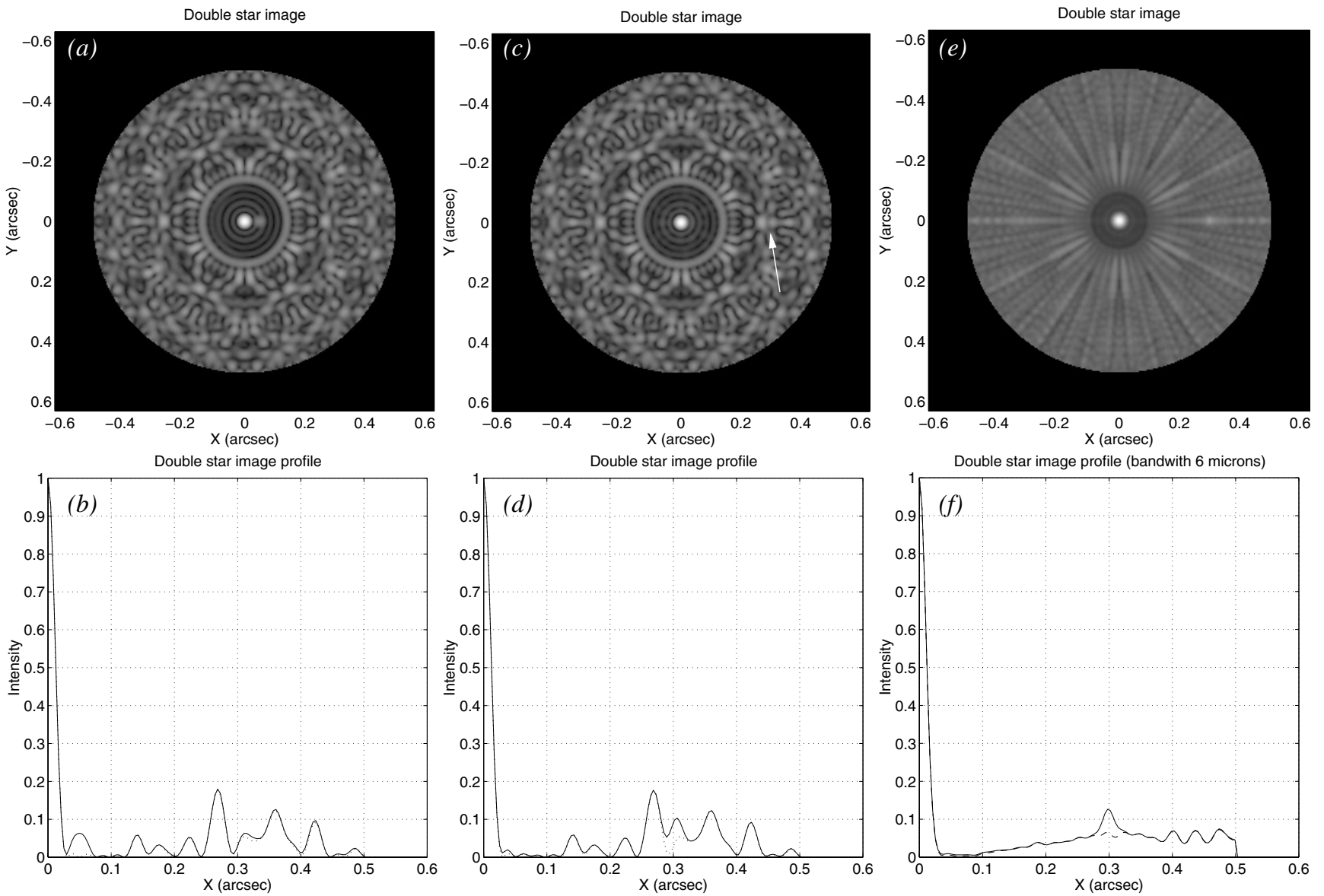

Fig. 10. Simulation of the direct image of binary systems in the pupil plane $P_{2}$. The instrumental configuration is described in the text. Interferometer resolution is 30 mas. a) Monochromatic image of a binary star separated by 50 mas, with a magnitude difference of 3 at a wavelength of $10 \mu \mathrm{m}$. b) Intensity cuts of the PSID (dashed line) and of the binary image (solid line), taken along the $x$-axis. c) Monochromatic image $(\lambda=10 \mu \mathrm{m})$ of a binary star of separation 300 mas and a magnitude difference of 3 . The companion is depicted by an arrow. d) Corresponding intensity profile (solid line) and PSID (dotted line). e) Polychromatic image $(\lambda=10 \mu \mathrm{m}, \Delta \lambda / \lambda=60 \%)$ of a binary of separation 300 mas and a magnitude difference of 3. f) Intensity cut of the corresponding image (solid line) and the PSID (dashed line) taken along the $x$-axis.

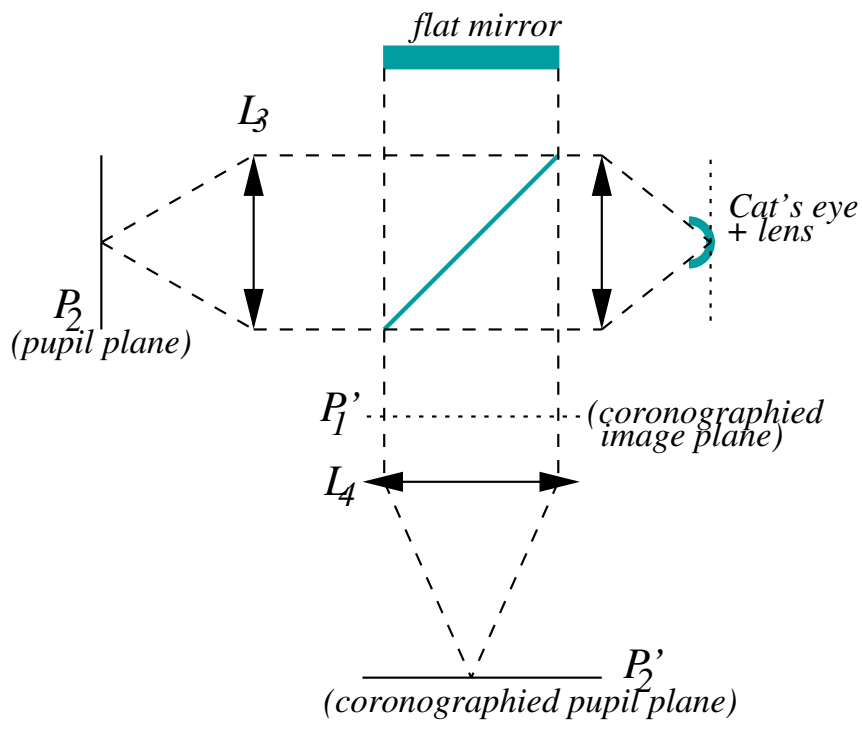

Fig. 11. Optical scheme of the AIC.

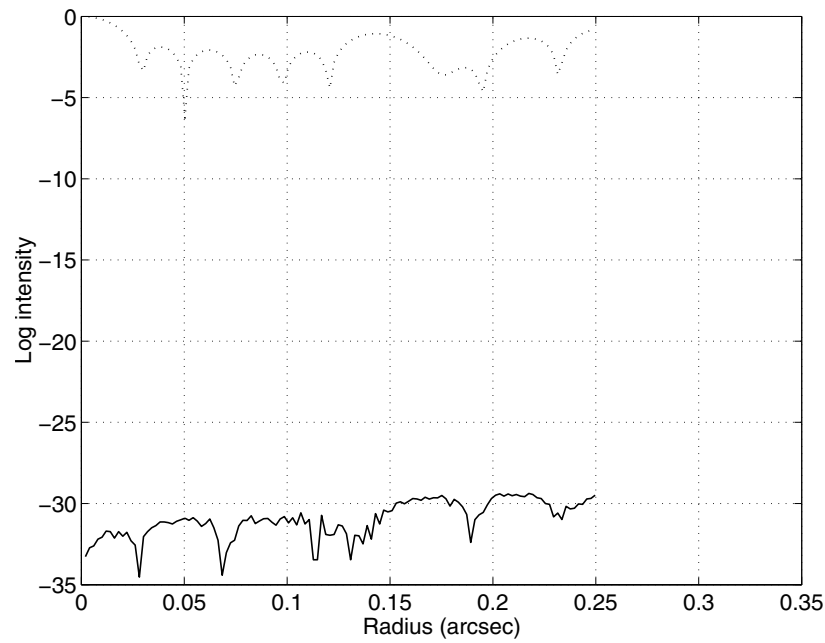

Fig. 12. Effect of coronography on the intensity of an on-axis star. Dotted line: intensity profile (taken along the $x$-axis) in the pupil plane $P_{2}$ without coronography. Full line: same but in the $P_{2}^{\prime}$ plane after having undergone the nulling effect through the AIC. 


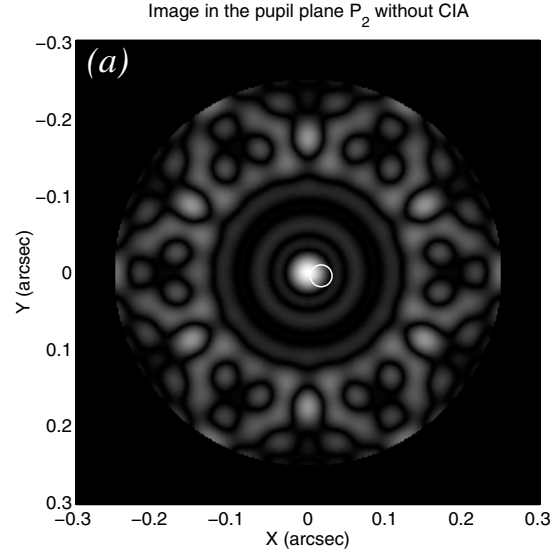

Image in the pupil plane $\mathrm{P}_{2}$ without $\mathrm{CIA}$

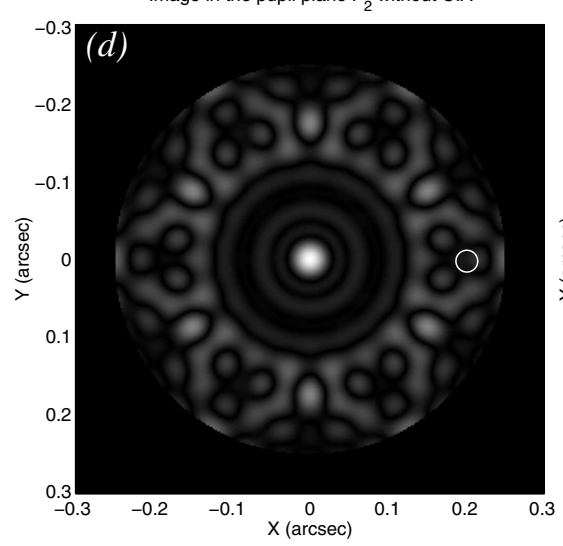

Image in the pupil plane $\mathrm{P}_{2}$ after $\mathrm{CIA}$

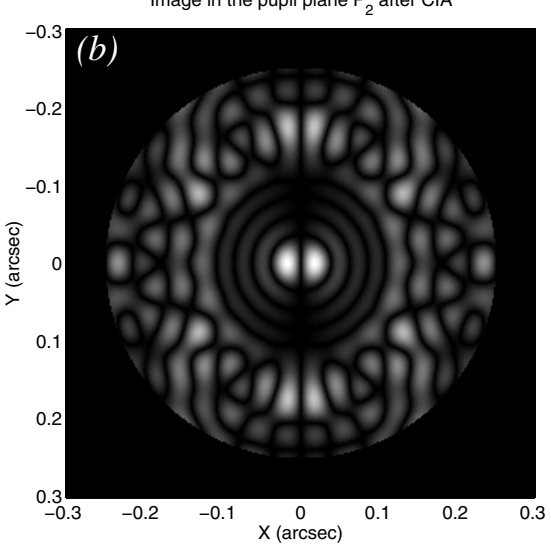

Image in the pupil plane $\mathrm{P}_{2}$ after CIA

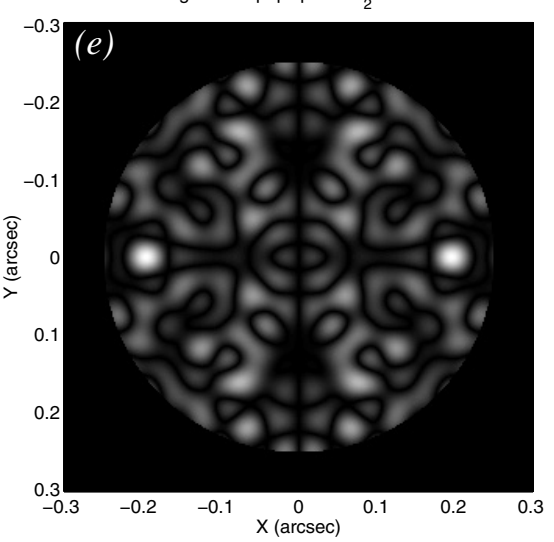

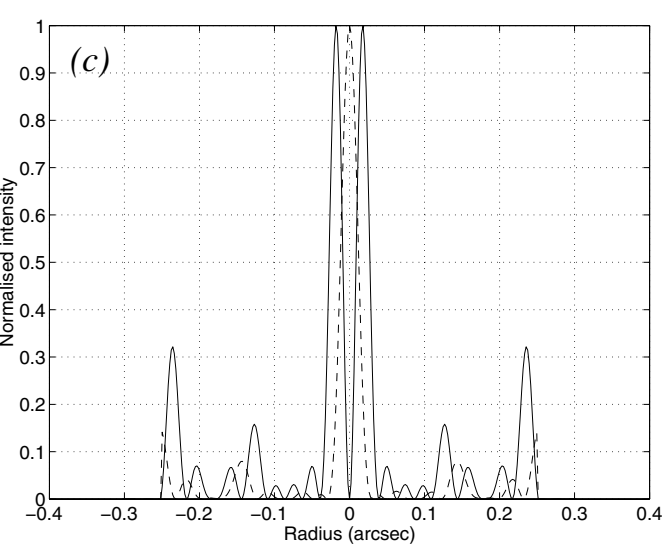

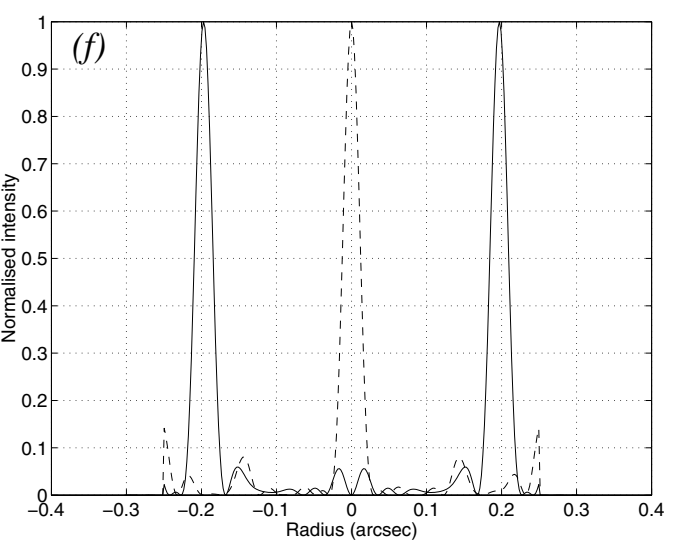

Fig. 13. Simulation of double stars monochromatic images $(\lambda=10 \mu \mathrm{m})$ in the pupil plane with and without coronography. a) Gray-level plot of the intensity without AIC for a magnitude difference of 5 and a separation of 10 mas. The companion (not visible) is depicted by the white circle. b) The same with AIC. c) Intensity profiles along the $x$-axis (solid line is with AIC, dotted line is without, maximum scaled to 1 for both curves). d)-f) are the same plots for a separation of 200 mas.

coherent energy concentrates in almost one pixel. Since the convolution relation subsists across the output stacked pupils any extended object will produce a one-to-one image inside that pupil, also optimum in terms of read-out and background noise. The shortcoming of IRAN however is that for imaging applications only a small central "clean-field" can be straightforwardly used. Even in this case deconvolution techniques could be applied to get rid of side-lobe noise although only half of the pupil would be usable for the FOV.

An interesting point that we shall exhaustively address in a next paper concerns the performances of the IRAN beamcombination versus the increasing number and size of primary telescopes forming the interferometric array. It is easy to understand that adding a new telescope to a array of $N$ existing telescopes increases by $N$ the cosine terms of modulation in the output stacked pupils. At the same time with the added telescope to the $N$ previous telescopes, supposed strictly identical, $(N+1) P$ photons will be modulated by these cosine terms. One can understand that if the modulation terms are completely non-redundant then the average modulated level of the photons at the pedestal of the central maximum of the IRAN PSID is $N+1$ times lower than the intensity of this maximum. This could intuitively be interpreted as followings: for photon-limited observations and a given collecting surface of the interferometer spread across a limited area, all things being equal, it is better to have more small telescopes than less large telescopes in terms of intensity concentration in the central peak for snap-shot imaging.

We also suggested two generic beam-combinations: one using bulky optics, the second a FO beam-combiner. In the first case the field of view has a constant photometric field of view whilst in a FO combination this field is multiplied by the monomode FO PSID. This does not hamper the imaging properties of IRAN but means that an ExPN for example would vanish photometrically with increasing distance from the center of the field. The FO combination is comparable with this respect to Labeyrie's densified pupil imaging where the field is modulated by the Airy figure of elementary telescopes.

The fact that IRAN produces a pseudo-Airy pattern inside the output stacked pupil arises the problem of central obscuration of the secondary mirror in a classical Cassegrain-coudé set-up of the telescopes. Thus the central zone of IRAN's field of view is "blind" to the on axis component of the source which is imaged by the interferometer. Off-axis primary telescope mirror combinations would therefore be preferable to apply IRAN, a solution which is also desirable for thermal IR interferometry to minimize background optics emission. On the other hand the convolution relation expressed in Eq. (17) depends on the $\gamma f_{2}$ densification factor. The validity of this convolution relation needs to be more thoroughly addressed for 
smoothly increasing values of $\gamma f_{2}$ from 1 , which corresponds to the Fizeau case, to larger values as outlined in Sect. 2.2, specially concerning the useful FOV for the detection of a an extra-solar planet in realistic conditions.

It remains that our technique works only if the telescopes were co-phased. In a coherenced array very probably the classical complex visibility using closure-phase and amplitude techniques are more applicable (Petrov et al 2000; Lopez 2003). The question of array co-phasing can be adressed by various techniques using for instance another spectral region (Bely et al. 1997) or more recent algorithms using spatio-spectral properties of densified pupils (Pedretti \& Labeyrie 1999).

Finally as already mentioned the field of view of IRAN is limited to the Airy disc of individual telescopes. At $10 \mu \mathrm{m}$ wavelength for instance, this limitation does not appear drastic. Considering a $2 \mathrm{~m}$ telescope for individual aperture, the Airy size is of the order of 1 arcsec. This size provides a field large enough to search for planets up to $100 \mathrm{AU}$ distance from their parent star.

\section{Conclusion}

We have presented a beam-combination technique with remarkable imaging properties for high dynamic imaging with diluted optical arrays. By construction the densified image and stacked-remapping technique from IRAN can be naturally combined with the Achromatic Interfero-Coronagraph (Gay \& Rabbia 1996), particularly suitable for coronographic imaging and detection of ExPNs compared to Labeyrie's densified pupil.

A number of questions remains open: the optimal beam combination, the effect of degrading co-phasing on the IRAN focal image, the imaging performances of IRAN after deconvolution for extended sources which exceed the central "clean-field" at the center of IRAN pupil and last but not least the formal definition of coronographic and/or nulling imaging of extended sources with IRAN. These question will be adressed in a next paper including a thorough comparison with the concept of DARWIN both in its nulling and imaging modes. The fore-coming studies and results will hopefully contribute to select the best beamcombination of next generation imaging optical arrays like the VLTI or extension of already opertaing imaging arrays like NPOI. However such arrays have not been originally designed for densified imaging since their PSF exhibits strong secondary interference maxima due to their sparse and irregular input array configuration. It is therefore mandatory that future synthesis arrays with a large number of primary telescopes (Vakili et al. 2004) involve an input baseline geometry which optimizes the PSID for its application to imaging/nulling schemes such as our proposed method.

Acknowledgements. We are grateful to N. Minec and J. Gay for constructive discussions on the basic ideas of our concept. The original manuscript has largely benefitted from the careful readings of $\mathrm{E}$. Thiébaut and W. Traub. We acknowledge A. Glindemann the referee, for his critical corrections and suggestions to improving the original manuscript. L. Abe benefits from a CNES post-doctoral fellowship and LUAN is supported by CNRS and UNSA.

\section{References}

Baudoz, P., Rabbia, Y., \& Gay, J. 2000a, A\&AS, 141, 319

Baudoz, P., Rabbia, Y., Gay, J., et al. 2000b, A\&AS, 145, 341

Beichman, C., Coulter, D., Lindensmith, C., \& Lawson, P. 2002, in Future Research Direction and Visions for Astronomy, Proc. SPIE, 4835, 115

Bely, P. Y., Haniff, C., Greenaway, A. H., et al. 1997, in Kilometric Space Interferometer, ESA-SP 96(97)

Bracewell, R. N. 1978, Nature, 274, 780

Chelli, A., \& Mariotti, J.-M. 1986, A\&A, 157, 372C

Gay, J., \& Rabbia, Y. 1996, C. R. Acad. Sci. Paris, 322 Série IIb, 265

Gillet, S., Riaud, P., Lardière, O., et al. 2003, A\&A, 400, 393

Gondoin, P., Absil, O., Fridlund, C. V. M., et al. 2003, in Interferometry for Optical Astronomy II, Proc. SPIE, 4838, 700

Gonsalves, R., \& Nisenson, P. 2003, PASP, 115, 706

Goodman, J. W. 1996, Introduction to Fourier Optics (Mc Graw-Hill Science)

Labeyrie, A., Le Coroller, H., Dejonghe, J., et al. 2003, in Interferometry in Space, Proc. SPIE, 4852, 236

Lopez, B., SF2A-2003, Bordeaux, France, June 16-20, 2003, ed. F. Combes, D. Barret, \& T. Contini (EDP Sciences), 155

Mariotti, J.-M., Coudé du Foresto, V., Perrin, G., Zhao, P., \& Léna, P. 1996, A\&AS, 116, 381

Mennesson, B., \& Mariotti, J.-M. 1997, Icarus, 128, 202

Michelson, A. A. 1920, ApJ, 51, 257

Pedretti, E., \& Labeyrie, A. 1999, A\&A, 137, 543

Pedretti, E., Labeyrie, A., Arnold, L., et al. 2000, A\&AS, 147, 285

Petrov, R., Malbet, F., Richili, A., et al. 2000, in Interferometry in Optical Astronomy, Proc. SPIE, 4006, 68

Rousselet-Perraut, K., Mourard, D., \& Vakili, F. 1997, Opt. Eng., 36, 980

Ruilier, C. 1999, Ph.D. Thesis, Observatoire de Paris

Soummer, R., Aime, C., \& Falloon, P. E. 2003, A\&A, 397, 1161

Tallon, M., \& Tallon-Bosc, I. 1992, A\&A, 253, 641

Traub, W. 1986, Appl. Opt., 25, 528

Vakili, F., Aristidi, E., et al. SF2A-2003, ed. F. Combes, D. Barret, \& T. Contini (EDP Sciences), 365 